\title{
The association of mortality with high temperatures in a temperate climate: England and Wales
}

\author{
Armstrong BG, Chalabi Z, Fenn B, Hajat S, Kovats S, Milojevic A, Wilkinson P.
}

London School of Hygiene \& Tropical Medicine, London.

Corresponding author: Ben Armstrong, London School of Hygiene \& Tropical Medicine, Keppel Street, London WC1E 7HT. Email: ben.armstrong@1shtm.ac.uk. Telephone: (+44) (0)207 927 2232

Key words: total mortality, time series, climate, heat.

7/12/2009 version: Word count: $3397-172-188=3037$

The Corresponding Author has the right to grant on behalf of all authors and does grant on behalf of all authors, an exclusive licence (or non-exclusive for government employees) on a worldwide basis to the BMJ Publishing Group Ltd and its Licensees to permit this article (if accepted) to be published in Journal of Epidemiology and Community Health and any other BMJPGL products to exploit all subsidiary rights, as set out in our licence (http://jech.bmj.com/ifora/licence.pdf).

Competing

Interest: None declared.

\section{What is already known:}

It is well known that high ambient temperatures are associated with increased mortality, even in temperate climates, but some important details remain unclear. In particular how heat-mortality associations (for example slopes and thresholds) vary by climate has previously been considered only qualitatively..

\section{What this paper adds}

English regions with hotter climates had higher threshold temperatures at which adverse effects started. Specifically, effects were well approximated by threshold-linear models that can be predicted from the region's climate $\left(93^{\text {rd }}\right.$ centile and mean summer temperature). On average mortality increased by $2.1 \%$ per degree Celsius above the $93^{\text {rd }}$ centile, but the increment was higher in hotter regions. This suggests partial adaption to hotter climates, relevant to estimating impacts from future climate change. 


\section{Abstract ( $n=239)$}

Background,

It is well known that high ambient temperatures are associated with increased mortality, even in temperate climates, but some important details are unclear. In particular how heat-mortality associations (for example slopes and thresholds) vary by climate has previously been considered only qualitatively.

\section{Methods}

We carried out an ecological time-series regression analysis of daily counts of all-cause mortality and ambient temperature in summers between 1993 and 2006 in the ten government regions, focusing on all-cause mortality and two-day mean temperature (lags 0 and 1).

Results

All regions showed evidence of increased risk on hottest days, but the specifics, in particular the threshold temperature at which adverse effects started, varied. Thresholds were at about the same centile temperatures (the $93^{\text {rd }}$, year-round) in all regions - hotter climates had higher threshold temperatures. Mean supra-threshold slope was $2.1 \% /{ }^{\circ} \mathrm{C}(95 \% \mathrm{CI}: 1.6,2.4)$, but regions with higher summer temperatures showed greater slopes, a pattern well-characterized by a linear model with mean summer temperature. These climate-based linear-threshold models capture most but not all the association; there was evidence for some nonlinearity above thresholds, with slope increasing at highest temperatures.

\section{Conclusion}

Effects of high daily summer temperatures on mortality in English regions are quite well approximated by threshold-linear models that can be predicted from the region's climate (93rd centile and mean summer temperature). It remains to be seen whether similar relationships fit other countries and climates or change over time, such as with climate change. 


\section{INTRODUCTION}

It is firmly established across many countries and areas that mortality increases at times of ambient temperatures that are unusually high for the area $[1,2]$. It is also widely found that the nature of the temperature-mortality relationship varies between places [1, 3-5]. In particular adverse effects of heat begin at lower temperatures in climates with lower summer temperatures, suggesting that populations adapt to their climate. [6] However, details of temperature-mortality associations (eg slope and threshold) need to be freshly identified for each place - a challenge for estimating burden of deaths due to heat and for setting alarm thresholds in heat watch warning systems. We have sought in this paper to fill this gap by proposing model in which the temperature-mortality association can be deduced from a region's climate. This has not previously been attempted.

Specifically, we characterized population temperature-mortality associations in summer in the ten government regions of England and Wales in the period 1993-2006. In particular, we aimed to identify climatic determinants which would explain any variation in these associations between regions and so allow parsimonious models that covered all of England and Wales. Downstream uses for this research include informing the Heatwave Plan for England [6], a warning system set up in 2004 by the British Government, and the estimation of impacts of climate change. We also sought to extend methodology for characterising the associations between mortality and temperature across populations with different climates, to facilitate similar research in other parts of the world.

\section{METHODS.}

We carried out an ecological time-series regression analysis of daily counts of all-cause mortality and measures of ambient temperature between 1993 and 2006 in the ten government regions of England and Wales [7].

Individual death record data were obtained from the ONS, including date of death and postcode of residence at time of death. The postcode was used to divide deaths by government region and date to make daily series of counts for each region. Here we consider counts of all deaths (including those from external causes).

We downloaded from the British Atmospheric Data Centre [8] all available monitoring station data on minimum and maximum dry-bulb temperature, from which the daily mean was estimated. From 9 am and 3pm dewpoint measurements we also estimated daily mean relative humidity and daily mean and maximum apparent temperature, an index designed to reflect the combined effect of humidity and temperature on heat stress [9]. The data were processed, imputing missing values the method of the AIRGENE study[10], to obtain population-weighted mean daily series for each region Further details are given in on-line annex 1.

The temperature series were highly correlated within regions (mean $\mathrm{r}=0.95$, range $0.94-0.98$ ) and station means varied little within region (mean $\mathrm{SD}=0.7{ }^{\circ} \mathrm{C}$ range $0.3-1.1$ ). Correlation between RH within regions was lower, on average 0.84 .

We did not attempt to estimate daily pollution series because variation of daily series from monitors within regions (typically comprising several different urban and rural areas) was expected to be too high to provide meaningful single regional series. 


\section{Statistical model}

Given the focus in these analyses on heat effects, to avoid complexities of simultaneous modelling cold effects we restricted analyses to summer, defined here as June-September (the hottest four months). Separate models were fit to each region. The region-specific models followed a fairly standard form for season-specific time series regression[1,11]. Our strategy was to fit and report a core confounder model decided a priori and explore the adequacy of this model and sensitivity of conclusions from fitting it through supplementary analyses. The models are summarised here and described formally in on-line annex 2.

All models assumed Poisson variation with scale overdispersion and residual auto-regression. The core confounder model comprised:

1. Within-summer seasonal variation: 4 degrees of freedom (df) natural cubic splines (NCS) in day-of-year (same for all years)

2. Trend in time over 11 years: linear and quadratic terms in time

3. 6 day-of week indicators

4. Relative humidity:3 df NCS for mean time lags 0-1 day (lag 0-1).

For sensitivity analyses (because routine incorporation was cumbersome), autoregression was allowed for using one of the lagged residual approach described by Brumback [12]. Terms were included to order three, which was the highest order with absolute partial autocorrelation of residuals above 0.05 in any region.

To describe region-specific temperature-mortality associations we initially used natural cubic splines fit to the daily maximum temperature over lags $0-1$, essentially equivalent to a distributed lag model constrained to have equal parameters for lags 0 and 1 [13]. Maximum temperature was chosen as the index providing best fit in exploratory analyses. Focus on lag 0-1 was chosen based on previous work in the UK $[14,15]$. We used default (equal quantile) knot placing and 4df - the number that maximised overall Akaike's Information Criterion (AIC). Splines were graphed as predicted mortality relative to that at the temperature at which mortality was minimum [16]. From the fitted spline models we also derived estimated temperature-specific slopes from the analytic first derivative of the spline basis terms.

Following description using the spline curves we fitted the simpler linear threshold (hockey-stick) model [13], by which mortality is not related to temperatures below a "threshold" and log-linearly related to temperatures above the threshold. Separate thresholds were initially fit to all regions. Distribution of thresholds and slopes across regions was then explored in a second stage analysis, graphically and using standard meta-analytic methods allowing for random effects between regions[17]. As will be described in the results section patterns of thresholds and slopes across regions suggested more parsimonious models with thresholds and slopes determined by regional climate.

In the threshold model deaths attributable to heat were estimated using the method of Bruzzi [18]. First daily attributable deaths were calculated from the number of deaths and the model-predicted relative risk due to heat on each day, then these were summed over all days, and divided by the total number of deaths.

To aid comparison of models, and in particular assess adequacy of the linear-threshold model we computed deviance and AIC corrected for over-dispersion (deviance $+2 p \varphi$, where $p=$ number of parameters and $\varphi$ is the estimated scale overdispersion) as well as overdispersion and various summaries of partial autocorrelation of residuals. Sensitivity analyses were carried out to explore 
robustness of key model features to model assumptions and the use of alternative temperature indices (minimum, mean, apparent).

\section{RESULTS}

There were on average 145 deaths per day in a region ranging from 72 in the NE to 200 in the SE. Figure 1 shows the regional temperature distributions (also summarized in Table 1) and fitted spline curves for temperature-mortality associations. In these and subsequent graphs and tables, the regions are shown in order of their mean summer daily maximum temperatures (which ranged from $18.4^{\circ} \mathrm{C}$ (NE) to $21.8^{\circ} \mathrm{C}$ (London)). 
Table 1 The conurbations: summer temperature distributions, number of deaths, increments in relative risk per degree under the linear-threshold model, and attributable deaths.

\begin{tabular}{|c|c|c|c|c|c|c|c|c|}
\hline \multirow[t]{2}{*}{ Region } & \multicolumn{3}{|c|}{$\begin{array}{c}\text { Distribution of summer daily maximum } \\
\text { temperature }\end{array}$} & \multicolumn{2}{|c|}{ Deaths } & \multirow{2}{*}{$\begin{array}{l}\text { Heat-mortality slope } \\
\text { ( } \% \text { per degree } \\
\text { above the threshold }))\end{array}$} & \multicolumn{2}{|c|}{$\begin{array}{l}\text { Deaths attributable } \\
\text { to heat }\end{array}$} \\
\hline & Mean & $(\min , \max )$ & $\begin{array}{l}93^{\text {rd }} \text { centile }^{1} \\
\text { (threshold) }\end{array}$ & Mean daily & Total & & percent & number \\
\hline North East & 18.4 & $(8.9,29.4)$ & 20.7 & 72.0 & 122,923 & $0.8(0.2,1.3)$ & 0.3 & 398 \\
\hline N West & 19.3 & $(11.5,32.0)$ & 21.7 & 189.0 & 322,820 & $1.3(1.0,1.6)$ & 0.6 & 2,067 \\
\hline $\begin{array}{l}\text { Yorkshire \& } \\
\text { Humberside }\end{array}$ & 19.5 & $(10.5,30.3)$ & 22.3 & 131.8 & 225,044 & $1.7(1.3,2.1)$ & 0.8 & 1,820 \\
\hline Wales & 19.6 & $(12.4,31.6)$ & 21.7 & 83.8 & 143,142 & $2.0(1.5,2.5)$ & 0.8 & 1,191 \\
\hline W Midlands & 20.3 & $(9.9,33.8)$ & 23.0 & 136.2 & 232,711 & $2.2(1.9,2.6)$ & 1.1 & 2,587 \\
\hline E Midlands & 20.3 & $(9.7,32.3)$ & 23.0 & 107.0 & 182,694 & $2.3(1.9,2.8)$ & 1.2 & 2,179 \\
\hline S West & 20.1 & $(12.3,30.9)$ & 22.3 & 136.1 & 232,520 & $2.1(1.7,2.5)$ & 0.9 & 1,986 \\
\hline S East & 21.0 & $(10.2,34.0)$ & 23.6 & 200.4 & 342,203 & $2.6(2.2,2.9)$ & 1.2 & 4,062 \\
\hline East & 21.1 & $(10.5,34.5)$ & 23.9 & 132.2 & 225,749 & $2.4(2.0,2.8)$ & 1.1 & 2,568 \\
\hline London & 21.8 & $(10.7,37.3)$ & 24.7 & 149.7 & 255,713 & $3.8(3.4,4.1)$ & 2.0 & 5,124 \\
\hline
\end{tabular}

Notes: 1. 93rd centile of year-round two-day mean temperature distribution. 2. DerSimonian and Laird estimate [19] 
Clear evidence of increased mortality at the highest temperatures was apparent for all regions except the cool North-East. The final panel in Figure 1 shows splines for all regions super-imposed on the same graph. Although not clearly discernable from the figure, the splines show subtle differences between regions beyond that attributable to the regions' different range of temperatures,. more clearly apparent if linear-threshold models are fit. For this thresholds were estimated by maximum likelihood (repeated calculation over a grid of values) in each region. The left panel of Figure 2 shows the thresholds and their likelihood-profile confidence interval (CI) by region sorted according to mean summer temperature. Although thresholds are estimated imprecisely, there is a clear pattern of higher thresholds in regions with higher mean temperatures (confirmed significant by meta-regression). The right panel of Figure 2 shows the same graph, but with thresholds expressed as the region-specific percentile of the (year-round) temperature distribution rather than as ${ }^{\circ} \mathrm{C}$. Heterogeneity remains but is reduced compared to that for thresholds as ${ }^{\circ} \mathrm{C}\left(\mathrm{I}^{2}=75 \%\right.$ Vs $\left.90 \%\right)$ and there is no longer any clear pattern by mean summer temperature. The best-fitting threshold by maximum likelihood (ML), if fixed to be at the same centile in each region, was at the $93^{\text {rd }}$ centile of (two-day mean) temperatures $\left(95 \%\right.$ CI $\left.92^{\text {nd }}-95^{\text {th }}\right)$.

We used this model (threshold at the regional $93^{\text {rd }}$ centile) to give a simple quantitative summary of the associations in each region that avoids having to rely on estimates made separately for each region. The region-specific thresholds $\left(93^{\text {rd }}\right.$ centiles $)$, the estimated slopes (increment in mortality per degree above the threshold) and heat-attributable deaths according to this model are given in Table 1. There was a strong association of slopes of these models with mean summer temperature (Figure 3), strongly statistically significant even if the obvious influential London region was excluded. This suggested possible a parsimonious model in which slopes were a function of the temperature distribution. In particular, we found that slopes were well predicted by regression on the mean summer temperature. (slope $=-.1284+0.0074=-\times($ mean summer temperature) $)$. We refer to this model as a climate-based threshold model.

To recapitulate, thresholds are related to the temperature distribution, approximating reasonably to the 93rd percentile in each region. With thresholds so placed, the supra-threshold slopes of the mortality-temperature relationships are still related to temperature, the steepest slopes occurring in the hottest cities. This suggests a climate-based threshold model, by which dependence of mortality on heat can be predicted from the climate of a city.

We additionally explored curvature in the supra-threshold temperature-mortality by adding a quadratic term (details not shown). We found that indeed this addition improved fit significantly. The direction of curvature varied between regions, but was predominantly positive (higher slopes at higher temperatures).

Table 2 shows deviance and AIC for the various models we have described above and some others, to allow identification of those that fit the data best. To aid comparison deviance and AIC are shown relative to a baseline of the model with all covariates but no temperature terms. Models best fitting for prediction are those with lowest (ie biggest negative) AIC. 
Table 2 Summary of fit of models (lag 01 mean temp) - all regions combined

Model description*

No temperature

Spline curves

$3 \mathrm{df}$

4 df

$5 \mathrm{df}$

$6 \mathrm{df}$

Threshold models

Linear: separate thresholds \& slopes

Linear: thresh at $\mathrm{P} 93 \%$; separate slopes

+quadratic terms

Linear: thresh at P93\%; slopes constrained df Deviance

160.0

$\begin{array}{lll}190.0 & -1730.3 & -1697.4 \\ \mathbf{2 0 0 . 0} & \mathbf{- 1 7 6 6 . 9} & \mathbf{- 1 7 1 2 . 2} \\ 210.0 & -1784.3 & -1707.4 \\ 220.0 & -1805.8 & -1706.7\end{array}$

$\begin{array}{lll}180.0 & -1674.8 & -1663.7\end{array}$

$171.0 \quad-1629.9 \quad-1638.0$

$181.0 \quad-1707.6 \quad-1694.7$

$163.0 \quad-1610.1 \quad-1635.6$

Alternative temperature measures (as 4df splines)

Max (ie core model)

Min

Mean

Mean apparent temperature

Max apparent temperature

Further models with 4 df temp spline:

add $4 \mathrm{df}$ spline for minimum temp

Knots placed at equal ${ }^{\circ} \mathrm{C}$

Lag 0

Lag 1

Lag 0-2

$\begin{array}{lrr}200.0 & -1766.9 & -1712.2 \\ 200.0 & -676.7 & -595.2 \\ 200.0 & -1522.4 & -1461.9 \\ 200.0 & -1418.7 & -1355.7 \\ 200.0 & -1742.7 & -1687.4\end{array}$

$240.0 \quad-1955.0 \quad-1814.7$

$200.0 \quad-1793.9 \quad-1739.9$

$200.0 \quad-1703.6 \quad-1647.0$

$200.0 \quad 1322.0 \quad 1256.8$

$200.0 \quad-1554.1 \quad-1494.5$

All models have the core covariate terms (day-of-year spline, linear-quadratic long term trend, dayof-week indicators, relative humidity spline) unless otherwise specified.

Deviance and AIC expressed relative to model with not temperature terms (20695.1 and 21089.0 resp)

The best fitting spline model (with $4 \mathrm{df}$ ) as graphed reduced deviance by 1766.9 and AIC by 1712.2. Some of the simpler models came close to this if number of parameters was penalised (ie by AIC) though did not quite match it. Although among linear-threshold models the one with region-specific thresholds fitted best, that with thresholds fixed at the 93rd centile came quite a close second by AIC. Constraining slopes to their value predicted by summer mean temperature improved fit slightly by AIC, because the loss in deviance terms was outweighed by the reduction in parameters, which are penalised by AIC. 
Comparison based on our prioritised model form (4 df spline for lag 0-1) confirmed daily maximum temperature to fit better than (in order of fit) maximum apparent, mean, mean apparent, or minimum temperatures. Adding spline terms for minimum temperature to the spline for maximum temperature (final block on table 2), improved fit. Replacing the default equal-quantile knots by equal-interval knots also improved fit slightly. Replacing the lag 0-1 mean of maximum temperatures by lag 0 or the lag 0-2 mean however reduced goodness of fit by AIC.

We explored sensitivity of key results to model assumptions. To simplify computing, we confined these to the linear-threshold model with threshold at the 93rd centile, and compared across models the mean slope and degree of heterogeneity between regions as indicated by the range of regionspecific slopes, on the grounds that most substantial sensitivity of other model features would be reflected in sensitivity in this simpler model. Though no model modification substantially changed results, the modification that was associated with greatest changes was that replacing the time terms (linear-quadratic trend and within-year spline terms) with elapsed month indicators (equivalent to a case-cross-over analysis with fixed strata).[20] In this model the mean supra-threshold slope was greater $\left(2.4 \%\right.$ per ${ }^{\circ} \mathrm{C}$ Vs 2.1$)$ ) and there was less variation between areas (range $2.6 \% \mathrm{Vs} 3.0 \%$ ). The pattern of higher slopes in hotter regions remained significant, however. Using the alternative temperature lags and indices shown on Table 2 changed the unit increments more - unsurprisingly since this changed the scale, but again not the pattern. More detailed results for the sensitivity analyses and model fit statistics are shown in on-line Tables E1 and E2.

\section{DISCUSSION}

Our finding that mortality is increased at higher temperatures, even in temperate climates such as England's, is not new. However, we have sought new precision in details of this relationship and patterns in its variation over regions. The following features were prominent in our findings:

- Threshold temperatures for heat effects to commence were at about the same centile temperatures (the 93rd) in all regions. Thus regions with higher summer temperatures on average had higher thresholds.

- However regions with higher summer temperatures showed greater increments in mortality per degree above the 93rd centile threshold (slopes), well-characterized by a linear model with mean summer temperature.

- Linear-threshold models capture most but not all the association. There was evidence for nonlinear impacts of temperature even above thresholds, with higher slopes at greater temperatures.

These findings suggested a parsimonious model for the pattern of variation in shape of associations between summer temperature and mortality, a climate-based threshold model with threshold following the 93rd centile and supra-threshold slope determined by a linear function of mean summer temperature. Such a parsimonious model has some advantages over region-specific estimates of associations: (i) it "borrows strength" from regions with information to more precisely estimate the association to allow better estimates for regions with less information; (ii) it allows estimation of heat impacts in a region not included in the study, since the relationship is determined by the temperature distribution,. (iii) it allows estimation of heat impacts under climate change, if adaptation is assumed such as that exhibited by the variation across regions (discussed below).

To achieve sufficient simplicity to characterize patterns of heat-mortality association across regions we have however put aside some detail. Perhaps most importantly, we have ignored evidence that the supra-threshold dependence of mortality on temperature is not linear (curved upwards). This 
can be accommodated in the above model at some complexity cost, for example by adding a quadratic term in supra-threshold temperature as described above.

For the same reason we have also not at this stage incorporated variables which might modify the heat impact. These include time in the season (some studies have shown great increment per degree earlier in the summer [1,21], duration of sustained heat (some studies have shown increased impact for sustained heat), personal vulnerability factors (many studies have shown older people at higher risk, some have shown lower socio-economic status to be at higher risk[22-25]). Similarly, we have not attempted to distinguish relationships for different causes of death, which are known to exhibit different degrees of sensitivity to heat $[1,21,26]$. Nor have we further evaluated the extent to which deaths caused by heat are displaced ("harvesting") [14, 27]. Most of these factors are similar over regions, so lack of allowance for them should not have substantially compromised our goal of characterising differences across regions, Deprivation is known to be greater in the (colder) North, but in England deprivation has not been found to predict vulnerability to heat. [22] Nevertheless, some residual confounding is likely and could operate differently across regions.

The model we propose is consistent with the observation in several studies that heat thresholds (or minimum mortality temperatures in all-year studies) are higher in hotter places [1, 4, 5, 26, 28, 29]. Honda [28] further found that minimum mortality temperatures across 47 prefectures in Japan were close to $80-85^{\text {th }}$ centile temperatures. Though the centile is different to the 93rd centile we found, the finding is similar in the sense that the a key feature of the relationship is at a constant centile across regions; the difference in centile could be due to use of all-year data and a spline model rather than summer data and a linear-threshold model. Baccini [1] also found higher heat slopes as well as higher thresholds in the hotter climates in Europe, though the threshold-climate relationship was not explored quantitatively. Anderson also found in US cities that relative risks across centiles of temperature distributions were less heterogeneous than were those for absolute temperatures, though here there was a tendency for lower heat slopes (RR across $99^{\text {th }}$ to 90 centile) in hotter climates. [3] This difference may be due to the substantial use of air conditioning in the US, in particular in its hotter cities.

Of the heat indices considered, maximum temperature fitted the data best, quite closely followed by maximum apparent temperature. There was some variation between regions in which index fitted best, with London for example favouring daily mean dry-bulb temperature. The relatively poor performance of minimum (night) temperature was somewhat surprising, but should not be taken to imply that these temperatures have no importance. When daily minimum temperature was added to a model addition to with daily maximum temperature, this improved model fit significantly, suggesting that minimum temperature has some importance independently of the maximum. The combination of maximum and minimum temperatures was also found important by Fouillet. [30] We have not in this paper sought to characterise this joint effect. Doing so is not trivial because of the high correlation of the two. There was little evidence that humidity was associated with mortality.

The implication in our climate-based threshold model of centile thresholds is that cities adapt to climate, since the proportion of days affected does not depend on climate. However, the implication of higher incremental risk per degree in hotter cities suggests that the adaptation is partial - there were higher attributable percentages of deaths in hotter cities (table 1). We hypothesized the possibility of using this partial adaptation model to estimate impacts of future climate change. This remains tentative, however. We have observed these patterns cross-sectionally not over time, and over a narrow climate range. Evidence from the USA suggests that factors little operating in England, perhaps air conditioning, can modify the dependence of heat vulnerability across climates. 
In summary, we have shown that effects of high daily summer temperatures on mortality in English regions are quite well approximated by threshold-linear models that can be predicted from the region's climate (93rd centile and mean summer temperature). It remains to be seen whether the relationship fits other countries and climates.

\section{Acknowledgements}

This study was funded by the UK Medical Research Council.

\section{REFERENCES}

1 Baccini M, Biggeri A, Accetta G, et al. Heat Effects on Mortality in 15 European Cities. Epidemiology 2008.

2 Basu R, Samet JM. Relation between elevated ambient temperature and mortality: a review of the epidemiologic evidence. Epidemiol Rev 2002;24:190-202.

3 Anderson BG, Bell ML. Weather-related mortality: how heat, cold, and heat waves affect mortality in the United States. Epidemiology 2009;20:205-13.

4 Curriero F, Heiner K, Samet J, et al. Temperature and mortality in eleven cities of the eastern United States. Am J Epidemiol 2002;155:80-7.

5 McMichael AJ, Wilkinson P, Kovats RS, et al. International study of temperature, heat and urban mortality: the 'ISOTHURM' project. Int J Epidemiol 2008;37:1121-31.

6 Department of Health. Heatwave Plan: 2008. London: DH 2008.

7 ONS. Map of Government Official Regions Office for National Statistics 2008.

8 BADC. British Atmospheric Data Centre. 2008.

9 Basu R, Ostro BD. A multicounty analysis identifying the populations vulnerable to mortality associated with high ambient temperature in California. Am J Epidemiol 2008;168:632-7. 10 Ruckerl R, Greven S, Ljungman P, et al. Air pollution and inflammation (interleukin-6, Creactive protein, fibrinogen) in myocardial infarction survivors. Environ Health Perspect 2007;115:1072-80.

11 Hajat S, Armstrong B, Baccini M, et al. Impact of high temperatures on mortality: is there an added heat wave effect? Epidemiology 2006;17:632-8.

12 Brumback B, Ryan L, Schwartz J, et al. Transitional regression models, with application to environmental time series. J Am Statist Ass 2000;95:16-27.

13 Armstrong B. Models for the relationship between ambient temperature and daily mortality. Epidemiology 2006;17:624-31.

14 Hajat S, Armstrong BG, Gouveia N, et al. Mortality Displacement of Heat-Related Deaths: A Comparison of Delhi, Sao Paulo, and London 2005.

15 Pattenden S, Nikiforov B, Armstrong BG. Mortality and temperature in Sofia and London. $J$ Epidemiol Community Health 2003;57:628-33.

16 Cao J, Valois M, Goldberg MS. An S-plus function to calculate relative risks for regression models using natural splines. Journal of Statistical Software 2005.

17 Sutton AJ, Abrams KR, Jones DR, et al. Methods for meta-analysis in medical research: Wiley Chichester 2000.

18 Bruzzi P, Green SB, Byar DP, et al. Estimating the population attributable risk for multiple risk factors using case-control data. Am J Epidemiol 1985;122:904-14.

19 DerSimonian R, Laird N. Meta-analysis in clinical trials. Control Clin Trials 1986;7:177-

88.

20 Farrington CP, Whitaker HJ. Semiparameteric analysis of case series data. Applied Statistics 2006;55:553-94. 
21 Hajat S, Kovats R, Atkinson R, et al. Impact of hot temperatures on death in London: a time series approach. J Epidemiol Community Health 2002;56:367-72.

22 Hajat S, Kovats RS, Lachowycz K. Heat-related and cold-related deaths in England and Wales: who is at risk? Occup Environ Med 2007;64:93-100.

23 Medina-Ramon M, Zanobetti A, Cavanagh DP, et al. Extreme temperatures and mortality: assessing effect modification by personal characteristics and specific cause of death in a multi-city case-only analysis. Environ Health Perspect 2006;114:1331-6.

24 O'Neill MS, Zanobetti A, Schwartz J. Disparities by race in heat-related mortality in four US cities: the role of air conditioning prevalence. J Urban Health 2005;82:191-7.

25 Schwartz J. Who is sensitive to extremes of temperature?: A case-only analysis.

Epidemiology 2005;16:67-72.

26 Braga AL, Zanobetti A, Schwartz J. The effect of weather on respiratory and cardiovascular deaths in 12 U.S. cities. Environ Health Perspect 2002;110:859-63.

27 Braga AL, Zanobetti A, Schwartz J. The time course of weather-related deaths. Epidemiology 2001;12:662-7.

28 Honda Y, Kabuto M, Ono M, et al. Determination of Optimum Daily Maximum

Temperature Using Climate Data. Environmental Health and Preventive Medicine 2007;12:209_ 16.

29 Kim H, Ha JS, Park J. High temperature, heat index, and mortality in 6 major cities in South Korea. Archives of Environmental and Occupational Health 2006;61:265-70.

30 Fouillet A, Rey G, Jougla E, et al. A predictive model relating daily fluctuations in summer temperatures and mortality rates. BMC Public Health 2007;7:114.

\section{Figure legends}

Figure 1 Temperature-mortality associations in each region (natural cubic splines with 4df; equal quantile knots)

Figure 2 Heat thresholds estimated by maximum likelihood separately for each region, as degrees and as percentiles, plotted against mean summer temperatures

Figure 3. Supra-threshold temperature-mortality slopes (\% per degrees) by mean of summer daily maximum temperatures (93-2006). 


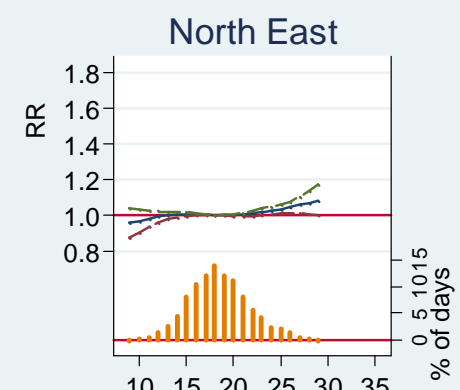

$10 \quad 15 \quad 20 \quad 25 \quad 30$

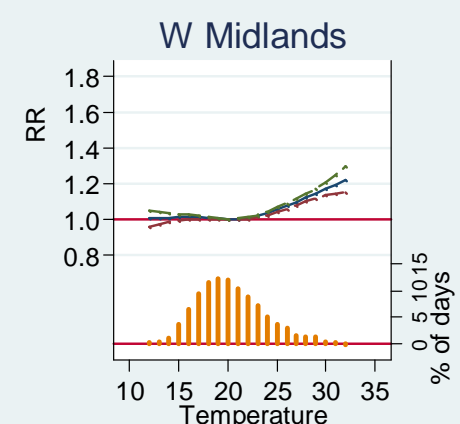

Temperature

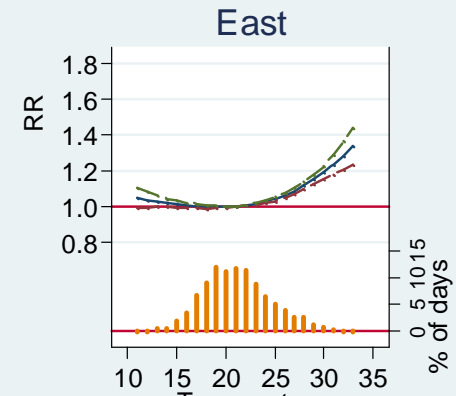

$10 \quad 15 \quad 20 \quad 25 \quad 30$
Temperature
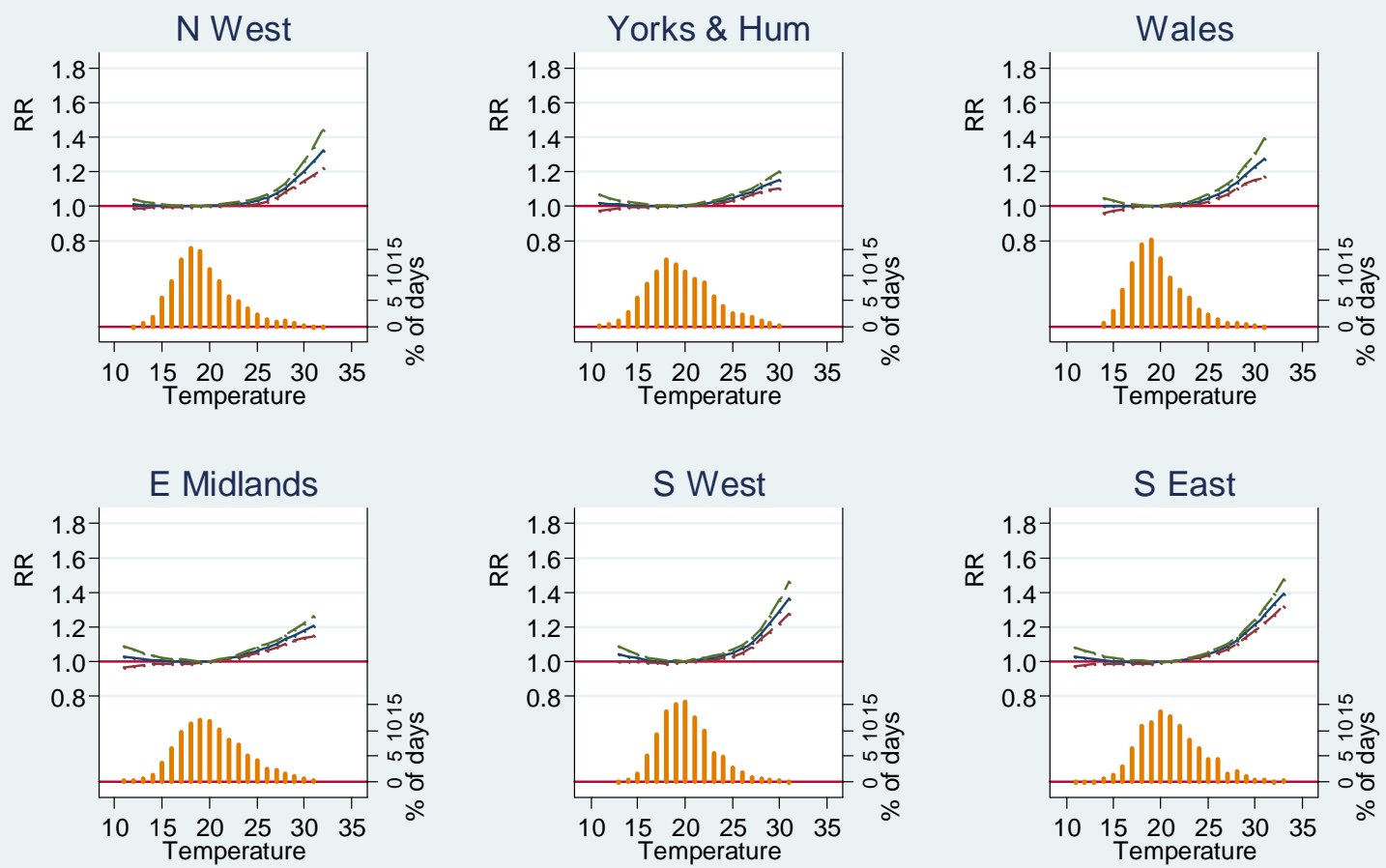

All
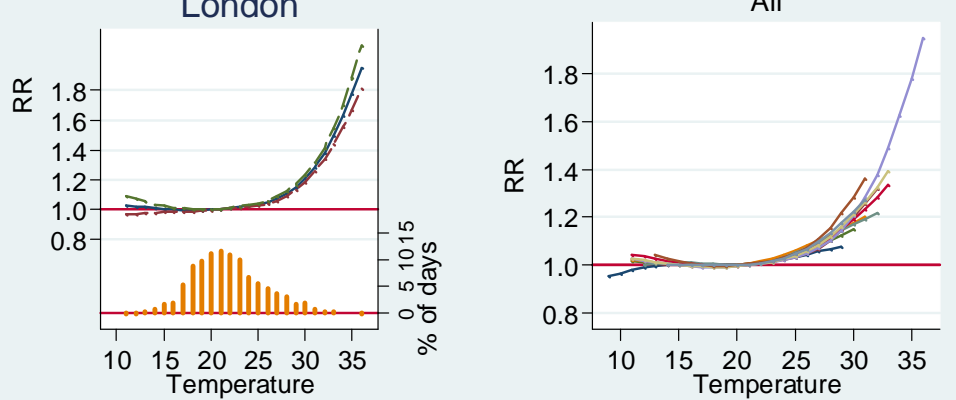

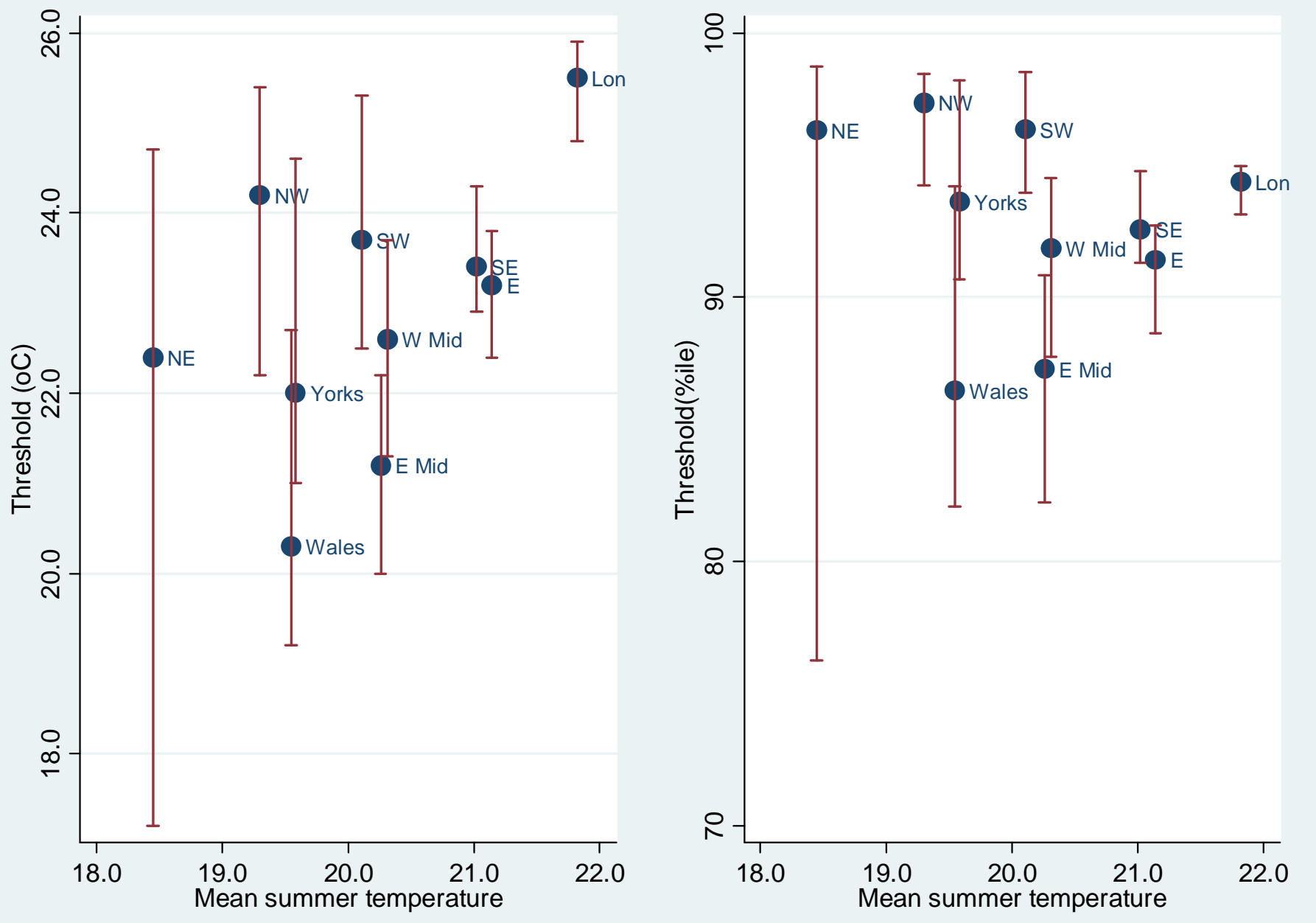


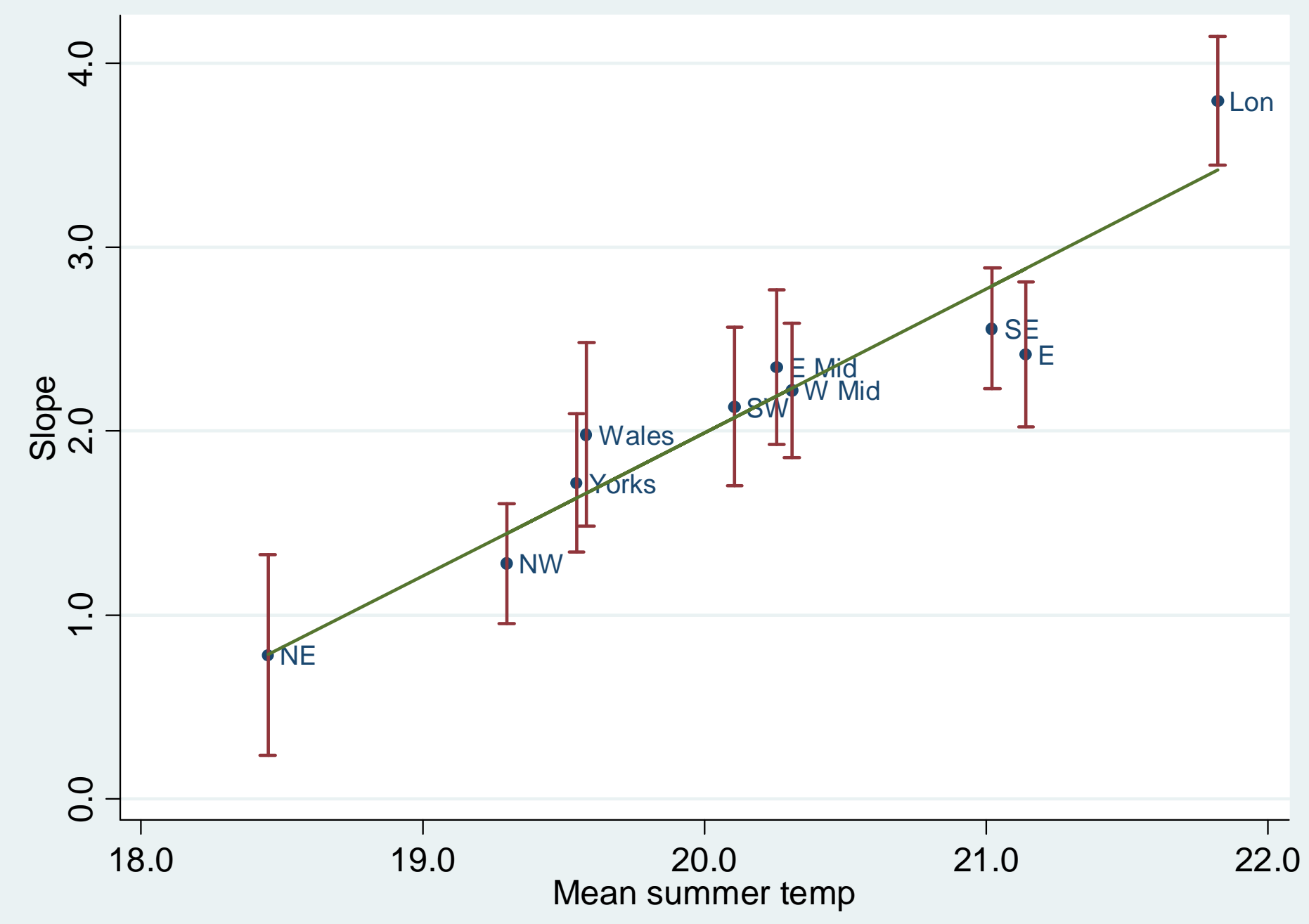

